Cellular Physiology
and Biochemistry and Biochemistry Published online: November 25, 2015

Accepted: October 14, 2015

This aricle is licensed under the tional License (CC BY-NC-ND) (he Creative Commons Attribution-NonCommercial-NoDerivatives 4.0 International License (CC BY-NC-ND) (http://www.karger.com/Services/OpenAccessLicense). Usage and distributio for commercial purposes as well as any distribution of modified material requires written permission.

\title{
Suppression of Development of Ankylosing Spondylitis Through Soluble Flt-1
}

\author{
Zhongxiang Yu ${ }^{a}$ Yuting Zhang ${ }^{b}$ Ningyang Gao Kuang Yong $^{a}$ \\ ${ }^{a}$ Department of Orthopaedics, Shu Guang Hospital affiliated to Shanghai Traditional Chinese Medical \\ University, Shanghai, 'Department of Orthopaedics and Trauma, Putuo District Center Hospital \\ affiliated to Shanghai Traditional Chinese Medical University, Shanghai, China
}

\section{Key Words}

Ankylosing spondylitis (AS) $\bullet$ sFlt- 1 • Monocytes $•$ Macrophages $\bullet$ Vessel permeability

\begin{abstract}
Background/Aims: Circulating monocytes/macrophages are origins of osteoclasts that mediate the development of ankylosing spondylitis (AS). Moreover, infiltrated macrophages facilitate the AS progression through production and secretion of pro-inflammatory cytokines. Thus, suppression of the recruitment of circulating monocytes/macrophages may be an effective AS treatment, which is, however, not available so far in clinic. Soluble fms-like tyrosine kinase-1 (sFlt-1) is a decoy receptor for vascular endothelial growth factor (VEGF) to compete with VEGF receptor (VEGFR2) for VEGF binding in endothelial cells, while its application in treating AS and effects on the recruitment of circulating monocytes/macrophages has not been reported before. Methods: We used a proteoglycan-induced arthritis (PGIA) mouse model for human AS. We injected sFlt-1 into the articular cavity and evaluated its effects on PGIA by incidence of arthritis, and clinical and pathological arthritis severity. We isolated and analyzed macrophages and endothelial cells in the articular cavity before and after treatment. Results: Injection of sFlt-1 significantly decreased the incidence and severity of PGIA in mice, and significantly reduced the number of infiltrated macrophages, possibly through reduction of vessel permeability, in a VEGFR2-dependent manner. Conclusion: Our data suggest that sFlt-1 may have a therapeutic effect on AS, resulting from suppression of VEGF signalingmediated recruitment of circulating monocytes/macrophages.
\end{abstract}

\section{Introduction}

Ankylosing spondylitis (AS) is a chronic inflammatory disease of the axial skeleton, with variable involvement of peripheral joints and non-articular structures. AS is one of the seronegative spondyloarthropathies and has a strong genetic predisposition. AS mainly

Z. Yu and Y. Zhang contributed equally to this work.

Zhongxiang Yu

Department of Orthopaedics, Shu Guang Hospital affiliated to Shanghai Traditional

Chinese Medical University, 528 Zhangheng Road, Shanghai 201203, (China)

E-Mail yuzhongxiang1@126.com pr E-Mail yuzhongxiang15@163.com 
affects joints in the spine and the sacroiliac joint in the pelvis, and may affect the whole spine in the severe cases [1-4]. AS can manifest as chronic back pain and a progressive spinal ankyloses, and often affect men of middle age [1-4].

The development of AS associates with major histocompatibility complex (MHC) class I since a majority of AS patients have human leukocyte antigen (HLA)-B27 (HLA-B27) gene [14]. Although the mechanisms of AS onset remain incompletely elucidated, the involvement of interactions among osteoclasts and inflammatory cells, as well as the production and secretion of a set of pro-inflammatory cytokines (e.g. TNF- $\alpha$, IL-6, and IL-10) have been acknowledged [1-4]. AS has been studied in various autoimmune models, including collageninduced arthritis [5-9] and proteoglycan-induced arthritis (PGIA) [10], both of which are mouse models for AS.

Macrophages have been found to predominate in biopsies from early spondyloarthritis inflammatory lesion. For example, AS macrophages have been found to produce an overabundance of IL-23 in response to TLR4 agonists in the infectious environment, thus predisposing to the development of inflammatory lesions. However, effective approaches that lead to inhibition of local infiltration of macrophages in AS are so far lacking.

Vascular endothelial growth factor (VEGF) family consists of the most important signal molecules that regulates angiogenesis, and is composed of six secreted proteins: VEGF-A, VEGF-C, VEGF-C, VEGF-D, VEGF-E and placental growth factor [11-15], among which VEGF-A is most potent for inducing endothelial cell proliferation and increasing endothelial cell permeability [16-19]. Soluble fms-like tyrosine kinase-1 (sFlt-1) is a well-known secretory decoy receptor for VEGF to compete with VEGF receptor (VEGFR2) for VEGF binding in endothelial cells, while it application in treating AS has not been reported before.

Here, we used a proteoglycan-induced arthritis (PGIA) mouse model for human AS. We injected sFlt-1 into the articular cavity and evaluated the effects on PGIA by incidence of arthritis, and clinical and pathological arthritis severity. We isolated and analyzed macrophages and endothelial cells in the articular cavity before and after treatment. We found that injection of sFlt-1 significantly decreased the incidence and severity of PGIA in mice, and significantly reduced the number of the macrophages, possibly through reduction of vessel permeability.

\section{Materials and Methods}

\section{Mouse handling}

All mouse experiments were approved by and performed according to the guidelines of the IACUC of Shu Guang Hospital affiliated to Shanghai Traditional Chinese Medical University. Only 12-week-old female Balb/c mice (Jackson lab, Bar Harbor, ME, USA) were used for in vivo experiments. The mice were kept in specific pathogen free (SPF) conditions.

Proteoglycan-induced arthritis (PGIA) model

Female Balb/c mice were i.p. injected with $100 \mu \mathrm{g}$ of cartilage proteoglycans (Sigma-Aldrich, St. Louis, MO, USA) on weeks 0,3 , and 6 . The first and third injections of proteoglycan were given in CFA (Difco, Detroit, MI, USA) and the second injection of proteoglycan was given in IFA (Difco) as has been previously described [20].

Intra-articular injection of sFlt-1

Intra-articular injection of sFlt-1 was performed as has been described before [20]. Mice were anesthetized with $2.5 \%$ isoflurane in $100 \%$ oxygen at a flow rate of $1 \mathrm{l} /$ minute. Hair was removed from both knee joints with a hair removal cream (Veet) to facilitate direct observation. The right knee joint of the mice was punctured once with a $30 \mathrm{G}$ needle below the patella to induce a hemorrhage. Subsequently a $3 \mu \mathrm{l}$ solution, containing $100 \mathrm{ng}$ recombinant mouse sFlt-1 (R\&D systems, Minneapolis, MN, USA) or vehicle only (phosphate-buffered saline containing $20 \mathrm{mmol} / \mathrm{l}$ Tris and $100 \mathrm{mmol} / \mathrm{l} \mathrm{NaCl}, \mathrm{pH}$ 7.5), was injected through a 33G needle in mice for each treatment. After these injections, the mice received acetaminophen via their drinking water to relieve pain until the end of the experiment. 


\section{Cellular Physiology Cell Physiol Biochem 2015;37:2135-2142

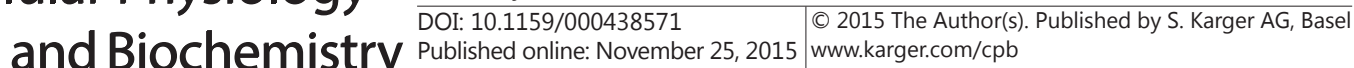 \\ Yu et al.: Suppression of AS Through sFlt-1}

\section{Assessment of arthritis}

Mice were scored blindly every other week for clinical signs of arthritis as has been described before [21]: 0 (no symptoms), 1 (redness and swelling in one toe), 2 (redness and swelling in more than one toe), 3 (toe stiffness), and 4 (deformity or ankle involvement). Hind paw forefeet were studied by histomorphology as has been described [21]: 0 (normal toe), 1 (acute inflammation including dactylitis), 2 (entheseal cell proliferation), 3 (cartilage formation), 4 (bone formation), and 5 (joint ankylosis). A cumulative score from all toes was calculated.

Quantification of Evans Blue (EB) extravasation in mouse joints

EB (Sigma-Aldrich) was i.v. injected to the mice at a dose of $200 \mu \mathrm{l}$ per mouse. After 30 minutes, mice were sacrificed and the mouse joint tissue was collected. Mouse tissue of $50 \mathrm{mg}$ was incubated with $500 \mu \mathrm{l}$ formamide to extract the extravasated EB. Optical density was measured at $610 \mathrm{~nm}$ and the measurements converted into ng dye extravasated per mg tissue.

Fluorescence-activated cell sorting (FACS) for macrophages and endothelial cells

Mouse joints were dissociated into single cells by $0.25 \%$ trypsin (Sigma-Aldrich), washed three times with vehicle solution, re-suspended in vehicle solution, labeled with either FITC-conjugated F4/80 antibody or PE-cy5-conjugated CD31 antibody (Becton-Dickinson Biosciences, San Jose, CA, USA), and then sorted either for F4/80+ macrophages or CD31+ endothelial cells. Data were analyzed and quantified using Flowjo software (Flowjo LLC, Ashland, OR, USA).

\section{RT- $q P C R$}

Total RNA was extracted from FAC-purified cells with RNeasy kit (Qiagen, Hilden, Germany). Complementary DNA (cDNA) synthesis was performed by reserve transcription. Quantitative PCR (RTqPCR) was performed in duplicates with QuantiTect SYBR Green PCR Kit (Qiagen). All primers were purchased from Qiagen. Quantification used ddCT method. Values of genes were first normalized against $\alpha$-tubulin, and then compared to experimental controls.

Statistics

GraphPad Prism software (GraphPad Software, Inc. La Jolla, CA, USA) was used for statistical analyses. Unpaired two-tailed Student $t$ test was applied for comparison between two groups from PGIA or PGIA plus sFlt-1. Data were represented as mean \pm SD and were considered significant if $p<0.05$.

\section{Results}

SFlt-1 inhibits development of PGIA in mice

Sixty Balb/c mice were immunized with proteoglycan through triple injections at week 0,3 and 6, as has been described [20]. Since 10 weeks, 30 mice received Intra-articular injection of sFlt-1 once per week for 4 weeks (week 10-13), while the other 30 mice received vehicle at same frequency. The mice were monitored for development of arthritis at 8, 9, $10,11,12,13$ and 14 weeks by evaluating the clinical severity and pathological severity (Fig. 1A). We found that sFlt-1-treated mice exhibited a significant decrease in the incidence of arthritis (Fig. 1B), a significant decrease in clinical severity (Fig. 1C), and a significant decrease in the pathological severity (Fig. 1D), compared to vehicle-injected mice. Thus, these data suggest that sFlt-1 inhibits development of PGIA in mice.

SFIt-1 significantly decreases the infiltrated macrophage number in PGIA

Since macrophage infiltration is regulated by VEGF signaling, and since macrophages play a key role in development of AS, not only by acting as a precursor for osteoclasts, but also by production and secretion of pro-inflammatory cytokines, we hypothesized that sFlt-1 may alter macrophage infiltration in PGIA. Thus, we dissociated articular cells at 14 weeks, and purified macrophages based on F4/80 by flow cytometry. We found that sFlt-1 significantly decreased the percentage of macrophages in the joints from PGIA mice, shown 
Fig. 1. sFlt-1 inhibits development of PGIA in mice. (A) Sixty Balb/c mice were immunized with proteoglycan through triple injections at week 0,3 and 6 , as has been described [20]. Since 10 weeks, 30 mice received Intra-articular injection of sFIT-1 once per week for 4 weeks (week 10-13), while the other 30 mice received vehicle at same frequency. The mice were monitored for development of arthritis at $8,9,10,11,12,13$ and 14 weeks by evaluating the clinical severity and pathological severity. (B) Incidence of arthritis. $n=30$.

(C) Clinical severity score.

(D) Pathological severity score. PG: proteoglycan. N $=30{ }^{*} \mathrm{p}<0.05$. Statistics: Unpaired two-tailed Student T test.

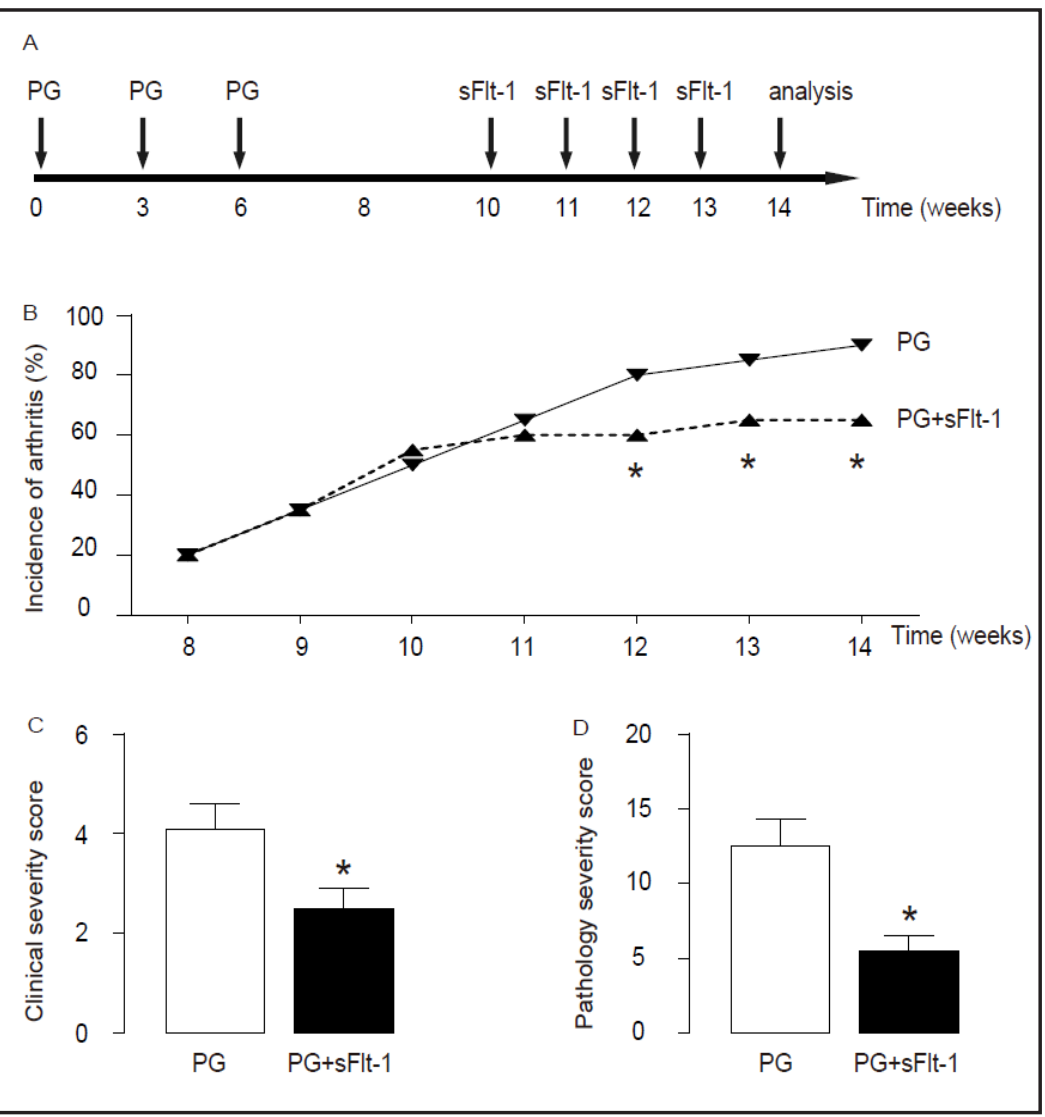

by representative flow charts (Fig. 2A), which was confirmed by F4/80 immunostaining of the sorted cells (Fig. 2B), and by quantification (Fig. 2C). We also analyzed the macrophage polarization with iNOS (a marker for M1 macrophages) and Arginase (a marker for M2 macrophages) in purified F4/80+ cells. We found a modest but significant increase in iNOS, and a modest but significant decrease in Arginase levels in macrophages from sFlt-1treated PGIA (Fig. 2D). These data suggest that sFlt-1 significantly decreases the infiltrated macrophage number in PGIA, and may modulate macrophage polarization to favor a M1 phenotype.

SFIt-1 decreases VEGFR2 levels in endothelial cells in PGIA

Since sFlt-1 competes with VEGFR2 on binding of VEGF in endothelial cells, which may affect the activation of endothelial cells upon VEGF challenge, we analyzed the changes in endothelial cells upon sFlt-1 treatment. Thus, we dissociated articular cells at 14 weeks, and purified endothelial cells based on CD31 by flow cytometry, shown by representative flow charts (Fig. 3A). We found that sFlt-1 did not significantly alter the percentage of endothelial cells in the joints from PGIA mice (Fig. 3B), although sFlt-1 indeed inhibited the activation of VEGFR2 on endothelial cells (Fig. 3C). These data suggest that sFlt-1 decreases VEGFR2 levels in endothelial cells in PGIA, but does not significantly increase the proliferation of endothelial cells in PGIA.

SFlt-1 inhibits increases in vessel permeability in PGIA mice

SinceVEGF may notonly induceendothelialcell proliferation, butalsoincreaseendothelial cell permeability, to alter infiltration of monocytes, we then used an Evans Blue (EB) assay to measure vessel permeability in PGIA mice. EB is a dye that binds albumin. Under physiologic conditions the endothelium is impermeable to albumin, so EB bound albumin remains within blood vessels. In pathologic conditions that promote increased vascular permeability 
Fig. 2. SFlt-1 significantly decreases the infiltrated macrophage number in PGIA. (A-C) We dissociated articular cells at 14 weeks, and purified macrophages based on F4/80 by flow cytometry. We found that sFlt-1 significantly decreased the percentage of macrophages in the joints from PGIA mice, shown by representative flow charts (A), which was confirmed by F4/80 immunostaining of the sorted cells (B), and by quantification (C). (D) RT-qPCR for iNOS and Arginase. $\mathrm{N}=30 .{ }^{*} \mathrm{p}<$ 0.05 . Statistics: Unpaired two-tailed Student $\mathrm{T}$ test. Scale bar is 20 $\mu \mathrm{m}$.

Fig. 3. sFlt-1 decreases VEGFR2 levels in endothelial cells in PGIA. (AC) We dissociated articular cells at 14 weeks, and purified endothelial cells based on CD31 by flow cytometry, shown by representative flow charts (A). We found that sFlt-1 did not significantly alter the percentage of endothelial cells in the joints from PGIA mice (B), although sFlt-1 indeed inhibited the activation of VEGFR2 on endothelial cells $(C)$. $N=30$. NS: non-significant. * $\mathrm{p}<$ 0.05. Statistics: Unpaired two-tailed Student T test.

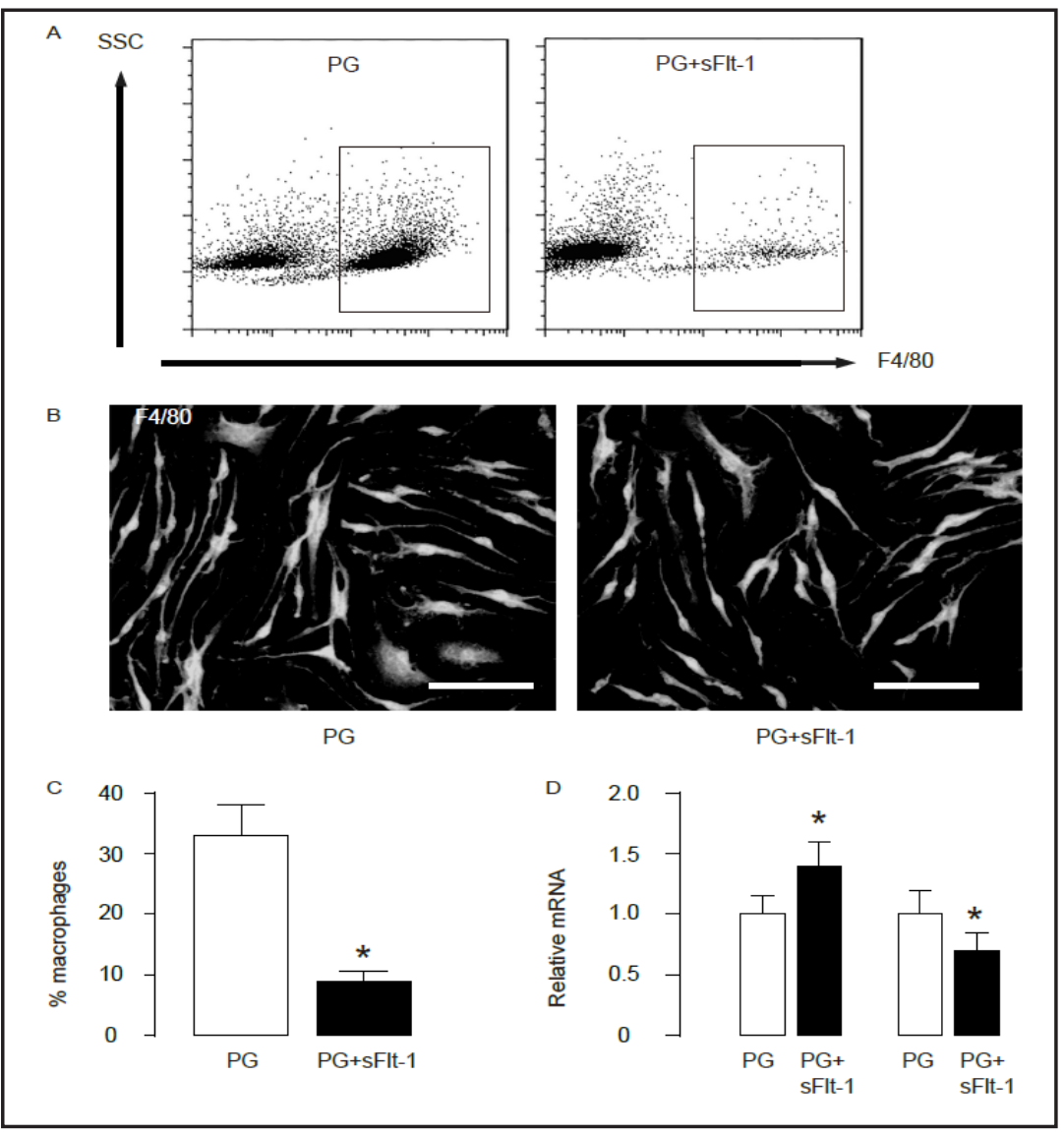

A

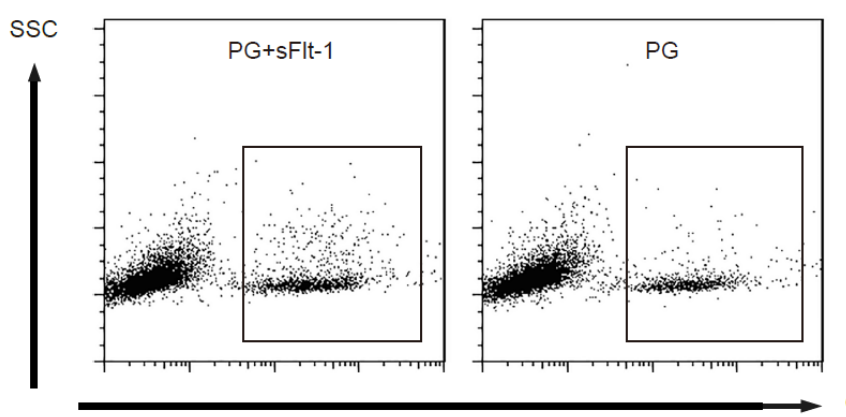

CD31
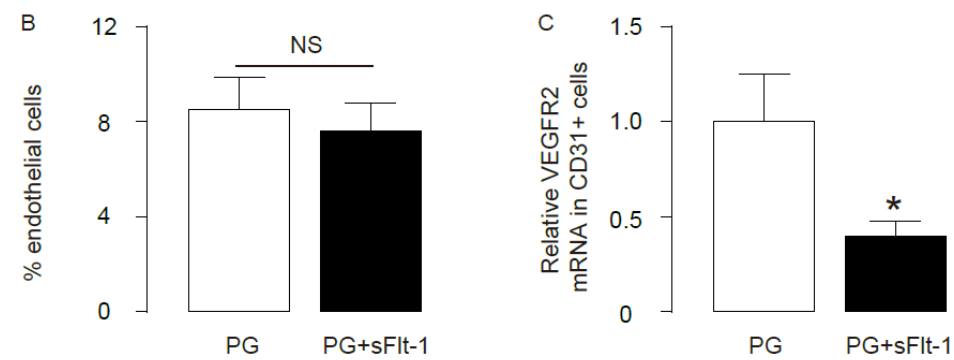

endothelial cells partially lose their close contacts and the endothelium becomes permeable to small proteins such as albumin. This condition allows for extravasation of EB in tissues. 


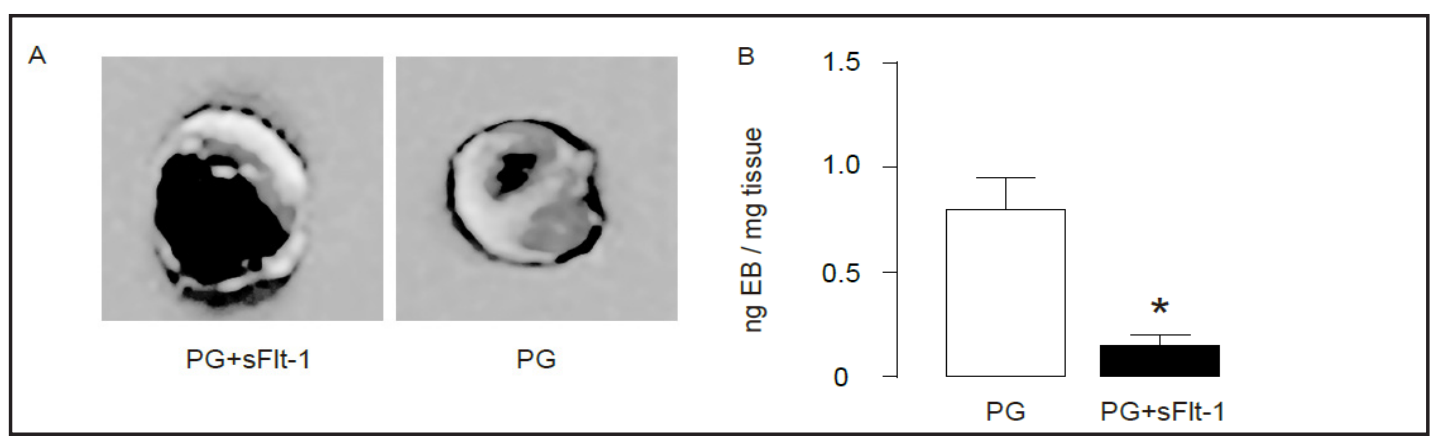

Fig. 4. sFlt-1 inhibits increases in vessel permeability in PGIA mice. (A-B) An Evans Blue (EB) assay to measure vessel permeability in PGIA mice. We found that the EB content in PGIA joints was significantly reduced by sFlt-1 treatment, shown by gross view of representative resected joints (A), and by quantification (B). $\mathrm{N}=30 .{ }^{*} \mathrm{p}<0.05$. Statistics: Unpaired two-tailed Student $\mathrm{T}$ test.

Fig. 5. A Schematic model. sFlt-1 may have a therapeutic effect on AS, resulting from suppression of VEGF signaling-mediated recruitment of monocytes/macrophages.

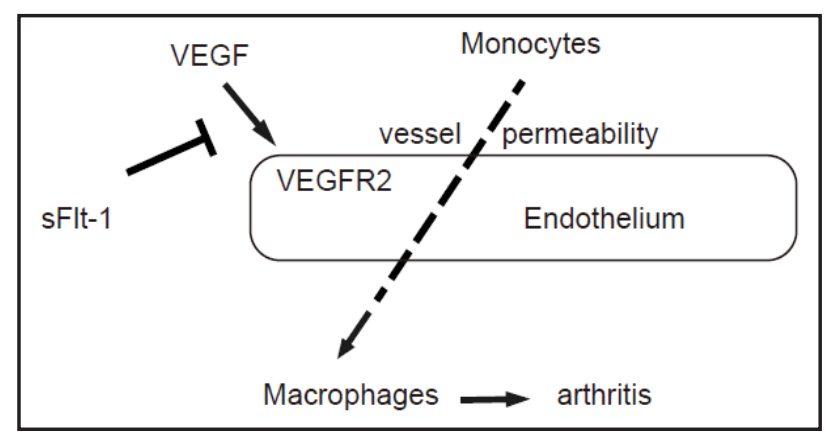

We found that the EB content in PGIA joints was significantly reduced by sFlt-1 treatment, shown by gross view of representative resected joints (Fig. 4A), and by quantification (Fig. 4B).Together, our data suggest that sFlt-1 may have a therapeutic effect on AS, resulting from suppression of VEGF signaling-mediated recruitment of monocytes/macrophages (Fig. 5).

\section{Discussion}

AS is a chronic, high-incident, inflammatory disease that mainly affects the sacroiliac peripheral joints, while also affects central main joints in severe cases [1-4]. Indeed, although AS typically involves the axial skeleton and the peripheral joints, extra-articular structures can be also affected [1-4]. The etiology and pathogenesis of AS are mainly discerned from studies at autopsy, biopsies of sacroiliac joints and sites of enthesitis, which highlights macrophages as a key player in the pathogenesis of AS.

Our hypothesis is based on studies from elsewhere. For example, Zhang et al. has shown that administration of a Chinese medicine after removal of the primary cancer significantly inhibited the growth of metastatic cancer cells in mouse liver. Further analyses showed that the soluble Flt-1 from this Chinese medicine neutralized the increased PLGF secreted by the metastatic cancer cells after primary cancer resection, and subsequently inhibited the cancer neovascularization to suppress the metastatic cancer growth [22]. This study encouraged us to use sFlt-1 to block vessel-dependent infiltration of monocytes/macrophages in AS. Animal models have been widely used for studying the mechanisms underlying AS molecular pathology [1-4]. There are different types of animal models for AS, including progressive ankyloses model, transgenic model, collagen-induced spondylitis and PGIA [1-4]. Here, we used PGIA model to study the underlying mechanisms of sFlt-1-mediated alleviation of arthritis severity.

Here, we injected sFlt-1 into the articular cavity of PGIA mice. We confirmed the therapeutic effects of sFlt-1 on PGIA by incidence of arthritis, clinical and pathological 


\section{Cellular Physiology Cell Physiol Biochem 2015;37:2135-2142 \begin{tabular}{l|l|l|} 
and BOI: 10.1159/000438571 2015 The Author(s). Published by S. Karger AG, Basel & $\begin{array}{l}\text { C) } 2015 \\
\text { www.karger.com/cpb }\end{array}$
\end{tabular} \\ Yu et al.: Suppression of AS Through sFlt-1}

arthritis severity, and thus proved the validity of the model. Then, we isolated macrophages from the articular tissue. Indeed, we found decreases in the percentage of macrophages by sFlt-1. These data suggest that sFlt-1 may affect the recruitment or proliferation of macrophages. Since sFlt-1 competes with VEGFR2 on VEGF binding, and VEGFR2 is mainly expressed by endothelial cells, we think that the direct effects of sFlt-1 may be on endothelial cells rather than macrophages. Then the changes in activities of endothelial cells may affect the macrophage recruitment.

However, sFlT-1 did not significantly change the endothelial cell number. Hence, the proliferation of endothelial cells is unlikely to be involved in the reduced infiltrated macrophages. Our next approach was to use a well-established EB method to test vascular permeability in vivo [23]. The assay is based on the fact that, under basal physiological conditions, albumin does not cross the endothelial barrier. EB is an azo dye with high affinity for albumin. When EB is injected in the blood stream of an experimental animal, and under physiologic conditions, it is expected to be retained within blood vessels. When a vascular permeability stimulus is added, blood vessels start to leak EB that is bound to albumin. Since vessel permeability is highly dependent on the age and weight of the animal, we thus used litter-mate animals in the same experiment to compare them.

Our study sheds new light on the therapeutic approaches of sFlt-1 on AS, and highlights that regulation of endothelia permeability may affect macrophage infiltration as a promising approach to treat AS.

\section{Disclosure Statement}

The authors have declared that no competing interests exist.

\section{References}

1 Dean LE, Jones GT, MacDonald AG, Downham C, Sturrock RD, Macfarlane GJ: Global prevalence of ankylosing spondylitis. Rheumatology (Oxford) 2014;53:650-657.

2 Golder V, Schachna L: Ankylosing spondylitis: An update. Aust Fam Physician 2013;42:780-784.

3 Colbert RA, Tran TM, Layh-Schmitt G: Hla-b27 misfolding and ankylosing spondylitis. Mol Immunol 2014;57:44-51.

4 Robinson PC, Brown MA: Genetics of ankylosing spondylitis. Mol Immunol 2014;57:2-11.

5 Myers LK, Tang B, Stuart JM, Kang AH: The role of il-4 in regulation of murine collagen-induced arthritis. Clin Immunol 2002;102:185-191.

6 Cottard V, Mulleman D, Bouille P, Mezzina M, Boissier MC, Bessis N: Adeno-associated virus-mediated delivery of il-4 prevents collagen-induced arthritis. Gene Ther 2000;7:1930-1939.

7 Lubberts E, Joosten LA, Chabaud M, van Den Bersselaar L, Oppers B, Coenen-De Roo CJ, Richards CD, Miossec P, van Den Berg WB: Il-4 gene therapy for collagen arthritis suppresses synovial il-17 and osteoprotegerin ligand and prevents bone erosion. J Clin Invest 2000;105:1697-1710.

8 Morita Y, Yang J, Gupta R, Shimizu K, Shelden EA, Endres J, Mule JJ, McDonagh KT, Fox DA: Dendritic cells genetically engineered to express il-4 inhibit murine collagen-induced arthritis. J Clin Invest 2001;107:1275-1284.

9 Yoshino S: Treatment with an anti-il-4 monoclonal antibody blocks suppression of collagen-induced arthritis in mice by oral administration of type ii collagen. J Immunol 1998;160:3067-3071.

10 Finnegan A, Grusby MJ, Kaplan CD, O'Neill SK, Eibel H, Koreny T, Czipri M, Mikecz K, Zhang J: Il-4 and il-12 regulate proteoglycan-induced arthritis through stat-dependent mechanisms. J Immunol 2002;169:33453352.

11 Ferrara N: Vascular endothelial growth factor. Arterioscler Thromb Vasc Biol 2009;29:789-791.

12 Otrock ZK, Makarem JA, Shamseddine AI: Vascular endothelial growth factor family of ligands and receptors: Review. Blood Cells Mol Dis 2007;38:258-268. 


\section{Cellular Physiology Cell Physiol Biochem 2015;37:2135-2142

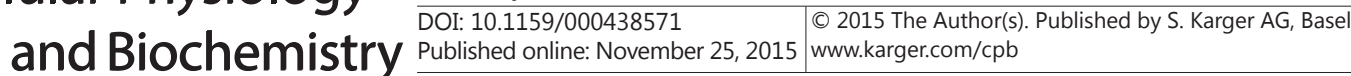 \\ Yu et al.: Suppression of AS Through sFlt-1}

13 Nieves BJ, D'Amore PA, Bryan BA: The function of vascular endothelial growth factor. Biofactors 2009;35:332-337.

14 Xiao X, Prasadan K, Guo P, El-Gohary Y, Fischbach S, Wiersch J, Gaffar I, Shiota C, Gittes GK: Pancreatic duct cells as a source of vegf in mice. Diabetologia 2014;57:991-1000.

15 Xiao X, Guo P, Chen Z, El-Gohary Y, Wiersch J, Gaffar I, Prasadan K, Shiota C, Gittes GK: Hypoglycemia reduces vascular endothelial growth factor a production by pancreatic beta cells as a regulator of beta cell mass. J Biol Chem 2013;288:8636-8646.

16 Ferrara N, Gerber HP, LeCouter J: The biology of vegf and its receptors. Nat Med 2003;9:669-676.

17 Carmeliet P, Jain RK: Molecular mechanisms and clinical applications of angiogenesis. Nature 2011;473:298-307.

18 Su JL, Yen CJ, Chen PS, Chuang SE, Hong CC, Kuo IH, Chen HY, Hung MC, Kuo ML: The role of the vegf-c/ vegfr-3 axis in cancer progression. Br J Cancer 2007;96:541-545.

19 Olofsson B, Jeltsch M, Eriksson U, Alitalo K: Current biology of vegf-b and vegf-c. Curr Opin Biotechnol 1999;10:528-535.

20 Lin S, Qiu M, Chen J: Il-4 modulates macrophage polarization in ankylosing spondylitis. Cell Physiol Biochem 2015;35:2213-2222.

21 Lories RJ, Derese I, Luyten FP: Modulation of bone morphogenetic protein signaling inhibits the onset and progression of ankylosing enthesitis. J Clin Invest 2005;115:1571-1579.

22 Zhang Y, Li A, Peng W, Sun J, Xu F, Xu J: Efficient inhibition of growth of metastatic cancer cells after resection of primary colorectal cancer by soluble flt-1. Tumour Biol 2015;36:7399-7407.

23 Radu M, Chernoff J: An in vivo assay to test blood vessel permeability. J Vis Exp 2013:e50062. 